Int. J. Morphol.,

31(1):115-120, 2013.

\title{
Lista de Verificación para el Reporte de Estudios Observacionales Descriptivos. Iniciativa MINCIR
}

\author{
Checklist for Reporting of Descriptive Observational Studies. MINCIR Initiative
}

\author{
Carlos Manterola \& Paula Astudillo
}

MANTEROLA, C. \& ASTUDILLO, P. Lista de verificación para el reporte de estudios observacionales descriptivos. Iniciativa MINCIR. Int. J. Morphol., 31(1):115-120, 2013.

RESUMEN: Los estudios observacionales descriptivos (EOD) constituyen al menos el $70 \%$ de los artículos publicados en las distintas revistas científicas del ámbito de la cirugía; y, a pesar de ello, no existen instrumentos para guiar a los escritores en el reporte de resultados, como tampoco para colaborar con los revisores con este tipo de artículos. El objetivo de este estudio es diseñar y construir un sistema de verificación para el reporte de resultados con EOD. Estudio bietápico con metodología cualitativa. En una primera etapa, se diseñó una propuesta, mediante la recopilación de ítems y dominios a partir de una extensa revisión de la literatura relacionada. En la segunda, se construyó una lista de verificación, aplicando reducción de ítems y dominios a través de un panel de 45 expertos en metodología de investigación, académicos clínicos, revisores y editores de revistas biomédicas; aplicando de este modo validez de fachada y de contenido. Los ítems y dominios incorporados a la lista fueron aquellos en los que se logró más de un $80 \%$ de acuerdo entre los participantes (36 de 45). Se generó de este modo un instrumento compuesto por 19 ítems, agrupados en 4 dominios. Se diseñó y construyó una lista de verificación que ayudará al reporte de resultados con EOD.

PALABRAS CLAVE: Estudios observacionales; Estudios descriptivos; Estudios longitudinales; Estudios de corte transversal; Series de casos; Reporte de casos.

\section{INTRODUCCIÓN}

Una de las acciones del proceso de investigación científica es la comunicación de resultados, cuya difusión a través de las revistas científicas es imprescindible. Estas, continúan siendo el canal más significativo de comunicación formal de los nuevos conocimientos que se generan y representan por ende, el vehículo habitual de divulgación de hallazgos, nuevos métodos y técnicas, etc. (Manterola, 2011).

Siguiendo esta línea de pensamiento; existe evidencia que gran parte de las preguntas que generan la investigación clínica originan con posterioridad estudios de tipo observacional (Glasziou et al., 2004, Alarcón \& Astudillo, 2007), y esto, debido a que los estudios observacionales juegan un rol relevante en la investigación sobre los beneficios y daños de las intervenciones (Black, 1996), detectar los efectos adversos infrecuentes o tardíos de los tratamientos (Papanikolaou et al., 2006), etc. Es así como, los estudios observacionales descriptivos (EOD), constituyen entre el
$70 \%$ y hasta el $90 \%$ de los artículos publicados en revistas científicas del ámbito de la cirugía y disciplinas afines; y, a pesar de ello, no existen instrumentos para guiar a los autores en el reporte de resultados, como tampoco para ayudar a los revisores y editores con este tipo de publicaciones (Funai et al., 2001; Scales et al., 2005; Pineda et al., 2005; Manterola et al., 2006a, 2006b; Manterola \& Grande, 2010).

Ahora bien, se sabe, que las recomendaciones sobre la forma de reportar los resultados de la investigación puede ayudar a mejorar la calidad; lo que se ha venido observando desde la aparición de la declaración CONSORT (Consolidated Standarts of Reporting Trials) para ensayos clínicos en 1996 (Moher et al., 2001); y otras iniciativas posteriores como STARD (Standards for Reporting of Diagnostic Accuracy), para estudios de pruebas diagnósticas (Bossuyt et al., 2003); MOOSE (Meta-analysis of observational studies in epidemiology), para el reporte de Meta-análisis de estudios observacionales (Stroup et al., 
2000); STROBE (Strengthening the Reporting of Observational Studies in Epidemiology), para el reporte de estudios observacionales, con especial hincapié en los estudios de cohortes y de casos y controles (von Elm et al., 2007); etc. Sin embargo, no existe una pauta para el reporte de EOD, a pesar de su alta prevalencia en las publicaciones biomédicas; razón por la cual, en 2011 publicamos el reporte preliminar de un estudio tendiente a generar una propuesta básica con este fin (Manterola \& Astudillo, 2011).

Los diseños incluidos entre los EOD son el reporte de casos, las series de casos (retrospectivas y prospectivas), los estudios de corte transversal, de pruebas diagnósticas y de concordancia; los estudios poblacionales y los correlacionales (Manterola, 2001, 2011; Burgos \& Manterola, 2010).

El objetivo de este estudio es diseñar y construir una lista de verificación para el reporte de resultados con EOD.

\section{MATERIAL Y MÉTODO}

Diseño. Estudio bietápico con estrategias cualitativas, para la generación de ítems y construcción del instrumento (Pope \& Mays, 1995; Jones \& Hunter, 1995). En la primera etapa se generaron los ítems y dominios; y en la segunda etapa se diseñó y construyó el instrumento.

Centro. El estudio fue realizado en el Departamento de Cirugía de la Universidad de La Frontera, en el período abril de 2009 y julio de 2012.

Metodología. En la primera etapa, se diseñó una propuesta, mediante la recopilación de ítems y dominios a partir de una extensa revisión de la literatura relacionada. En la segunda, se construyó el instrumento, aplicando reducción de ítems y dominios a través de un panel de expertos, a quienes se consultó a través de un cuestionario semiestructurado y autoadministrado, compuesto por 25 ítems provenientes de la literatura; a los que los expertos podían agregar otros según su experiencia o particular visión de la situación. De este modo, se generó la validación de contenido, tanto de fachada como de muestreo; asegurando así, que el instrumento contiene ítems representativos de todas las áreas que definen el concepto o constructo en estudio (el que quedó a resguardo, por una exhaustiva revisión de la literatura, la experiencia del grupo de investigación y el panel de expertos).

Participantes. El panel de expertos estuvo compuesto por 4 epidemiólogos clínicos, 5 estadísticos; 28 académicos clínicos y miembros de diversos paneles de revisores de revis- tas biomédicas y 8 editores de revistas biomédicas $(n=45)$. La mediana de edad de éstos fue de 52 años (35 a 70 años). Treinta y nueve son de género masculino $(86,7 \%)$ y 19 de centros internacionales $(42,2 \%$; 11 de centros sudamericanos, 4 de centros europeos y 4 de centros norteamericanos). Sus profesiones de origen son: médicos especialistas (33), bioestadísticos (5), enfermeras (5), odontólogo (1) y kinesiólogo (1); con una mediana de tiempo de experiencia profesional de 28 años (5 a 47 años), 38 de los cuales $(84,4 \%)$, se desempeñan en centros universitarios.

Recolección de datos. La segunda etapa se desarrolló utilizando una estrategia cualitativa mediante técnica Delphi (Powell, 2003; Kennedy, 2004; Price, 2005). Ésta, se aplicó en fases: se seleccionaron los expertos, se les formuló la pregunta de investigación y se estructuraron las respuestas a modo de un cuestionario para consultarles respecto de su acuerdo con los puntos señalados sobre los elementos que se consideraban fundamentales; en este caso, los ítems y dominios que configurarían la pauta de reporte. Con cada retroalimentación-respuesta a partir de los cuestionarios anteriores los expertos pudieron establecer un consenso general.

Plan de análisis. Después de una extensa revisión bibliográfica sobre el tema, se verificó que no existe consenso plasmado en una pauta para el reporte de EOD. Se construyó la pregunta de investigación (primer borrador); esta, se planteó a los expertos y ellos respondieron de forma individual, privada y anónima; posterior a lo cual se analizaron las respuestas, las que se resumieron y convirtieron en ítems y dominios. De este modo, cada miembro del panel de expertos evaluó cada ítem de acuerdo a sus conocimientos y experiencia (segundo borrador). Posteriormente, se generó una base de datos ad-hoc para el almacenamiento de los datos, los que fueron analizados en un programa Stata 10.0/ SE ®. Después de realizar un análisis exploratorio de los datos, se aplicó estadística descriptiva con cálculo de porcentajes, medianas y valores extremos.

\section{RESULTADOS}

Los ítems generados en la primera etapa (25), se agruparon inicialmente en 5 dominios: título, resumen, introducción, metodología, resultados, discusión y conclusiones. Al aplicar el cuestionario al panel de expertos, se generó una reducción de ítems y dominios.

Los ítems y dominios incorporados al instrumento final fueron aquellos en los que se logró más de un $80 \%$ de acuerdo entre los participantes (en 36 de 45). A éstos, se adicionaron aquellos ítems propuestos por los integrantes 
del panel, cuando al menos dos de ellos coincidieron en la relevancia de incorporarlos.

De este modo, se construyó un instrumento que quedó compuesto por 19 ítems (problema en estudio, objetivos, diseño del estudio, lugar donde se desarrolló la investigación, características de los participantes, criterios de inclusión y exclusión, tipo de muestreo utilizado, variables estudiadas, seguimiento realizado, estadísticas utilizadas, principios éticos observados, descripción general de la muestra estudiada, análisis de grupos y subgrupos, otros análisis (si corresponde), novedad de la propuesta que representa el artículo, comentarios de los resultados obtenidos, limitaciones del estudio y conclusión (es) si es procedente); agrupados en 4 dominios: Introducción, metodología, resultados y discusión.

Se procedió luego a la última etapa Delphi, la socialización de los resultados, momento en el que los expertos revisaron los ítems, dominios y sus respectivos comentarios aclaratorios; llegando al instrumento final que se esquematiza en la Tabla I.

Tabla I. Instrumento generado. Lista de verificación para el reporte de estudios observacionales descriptivos. Iniciativa MINCIR.

\begin{tabular}{|c|c|c|}
\hline Dominio & Ítem & Pregunta clave \\
\hline \multirow[t]{7}{*}{ Introducción } & Problema en estudio & $\begin{array}{l}\text { ¿Desarrolla un enfoque general del problema en estudio, de la información } \\
\text { científica disponible y la justificación de la investigación que se está } \\
\text { reportando? }\end{array}$ \\
\hline & Objetivos & ¿Se plantean objetivos claros y precisos? \\
\hline & Diseño del estudio & $\begin{array}{l}\text { ¿Menciona el diseño de estudio utilizado? Por ejemplo: "Se trata de una serie } \\
\text { de cas os de carácter retrospectivo...", o "de un estudio de corte tran sversal". }\end{array}$ \\
\hline & $\begin{array}{l}\text { Lugar donde se desarrolló la } \\
\text { investigación }\end{array}$ & $\begin{array}{l}\text { ¿Describe el escenario, lugares, y fechas correspondientes; incluidos la } \\
\text { eventual exposición, el seguimiento y la recopilación de datos? }\end{array}$ \\
\hline & Participantes & ¿Indica el número de sujetos estudiados o el tamaño de la muestra utilizada? \\
\hline & Criterios de inclusión & ¿Señala los criterios de inclusión de la población estudiada? \\
\hline & Criterios de exclusión & $\begin{array}{l}\text { ¿Cita los criterios de exclusión de la población estudiada? (recordar que éstos } \\
\text { no son lo contrario de los criterios de inclusión). }\end{array}$ \\
\hline \multirow[t]{6}{*}{ Metodología } & Muestreo & ¿Indica el tipo de muestreo utilizado? (cuando corresponda). \\
\hline & Variables & $\begin{array}{l}\text { ¿Define claramente las variables estudiadas? Idealmente la variable principal } \\
\text { o de resultado y "otras variables de interés". Si corresponde, señalar quién, } \\
\text { cómo, con qué y cuándo midió. }\end{array}$ \\
\hline & Seguimiento & $\begin{array}{l}\text { ¿Menciona el tiempo de observación o de seguimiento de los sujetos en } \\
\text { estudio? (según corresponda). }\end{array}$ \\
\hline & Estadísticas & $\begin{array}{l}\text { ¿Señala las herramientas estadísticas utilizadas? Referirse a la s estadísticas } \\
\text { desc riptivas y analíticas que se emplearon (según corresponda). }\end{array}$ \\
\hline & Principios éticos & ¿Indica los principios éticos involucrados? \\
\hline & Participantes & $\begin{array}{l}\text { ¿Describe de forma general la muestra estudiada? Recomendable utilizar } \\
\text { estadística descriptiva. }\end{array}$ \\
\hline \multirow[t]{3}{*}{ Result ados } & Análisis de grupos y subgrupos & $\begin{array}{l}\text { ¿Aplica estadística analítica y c omparación de grupos y subgrupos? (si } \\
\text { corresponde). }\end{array}$ \\
\hline & Otros análisis & $\begin{array}{l}\text { ¿Emplea otro tipo de análisis estadísticos? Por ejemplo: análisis de } \\
\text { supervivencia, ajustes por factores de confusión y su precisión estimando los } \\
\text { intervalos de confianza del } 95 \% \text { (según corresponda). }\end{array}$ \\
\hline & Novedad de la propuesta & $\begin{array}{l}\text { ¿Discute acerca de los aspectos novedosos del estudio que se presenta? Por } \\
\text { ejemplo: El objetivo, el diseño, las características de la población, la } \\
\text { intervención, la medición de los resultados, etc. }\end{array}$ \\
\hline \multirow[t]{3}{*}{ Discusión } & $\begin{array}{l}\text { Comentarios respecto de los } \\
\text { resultados }\end{array}$ & $\begin{array}{l}\text { ¿Comenta e interpreta los resultados obtenidos en relación al conocimiento } \\
\text { existente y resultados de estudios similares? }\end{array}$ \\
\hline & Limitaciones del estudio & ¿Expone las limitaciones del estudio y los potenciales sesgos existentes en él? \\
\hline & Conclusión (es) & $\begin{array}{l}\text { ¿Plantea una conclusión? Sólo para aquellos estudios en los que se pueda } \\
\text { plantear. Es decir, si corresponde según objetivo, diseño y resultados } \\
\text { observados. }\end{array}$ \\
\hline
\end{tabular}




\section{DISCUSIÓN}

En general, se piensa que sólo los ensayos clínicos (EC), son estudios primarios idóneos para generar evidencia; y hay quienes incluso rechazan categóricamente la posibilidad de que los EOD sirvan para ello. Esto, a consecuencia que en los EOD no se controla la asignación del paciente a un determinado tratamiento o intervención, transformándose el investigador en un mero observador, descriptor y relator de lo que ocurre. No obstante ello, existen grupos que piensan que las restricciones impuestas a los pacientes incluidos en un EC a menudo originan que éstos difieran de las características del paciente habitual en la práctica clínica; además los centros participantes suelen ser centros específicos, de referencia o universitarios, también con características especiales respecto al colectivo. Por ello se estima que no siempre son generalizables las conclusiones obtenidas a partir de EC.

Sin embargo, parece más sensato adoptar una posición más conservadora, admitiendo por una parte que los EC constituyen la mejor herramienta metodológica a utilizar cuando de tratamiento y prevención se trata; y por otra, que los EOD bien desarrollados y con resultados reportados de forma adecuada, también constituyen herramientas metodológicas apropiadas para generar evidencia (Black).

Así, se puede comprender que lo que motiva a conducir EOD es: describir manifestaciones inusuales de un evento de interés o el efecto de una exposición que no puede ser asignada de forma aleatoria (por ejemplo someterse a una cirugía, a un procedimiento invasivo, etc.); describir enfermedades raras o eventos de interés poco usuales; generar conocimiento de la historia natural o del curso clínico de una entidad nosológica; obtener frecuencias de las diversas variables de un evento de interés; permitir la formulación de hipótesis de posibles factores de riesgo; realizar vigilancia epidemiológica; estudiar la validación externa de la aplicación de una intervención (verificar que se cumplan las condiciones de eficacia y tolerancia en la práctica habitual), de la adhesión a una intervención (grado de cumplimiento del tratamiento por parte de los participantes), etc. (Manterola, 2001, 2001).

A pesar de ello, se ha de comprender con claridad que este tipo de estudio tienen serias limitaciones, entre las que destacan: que representan la experiencia limitada a una sola persona o grupo de investigación, lo que redunda en la subjetividad personal de quien (es) reporta (n), lo que puede generar sesgos de medición; los inconvenientes generados por sesgos de reporte, relacionados con la selección y referencia; considerar que la presencia de un factor de riesgo puede ser efecto del azar; que como la observación se inicia en diferentes puntos del curso de la enfermedad o evento de interés en estudio, resulta difícil ser enfático respecto de asociaciones temporales. En resumen, se ha de tener presente, que los EOD no constituyen evidencia sólida como base para alterar la práctica clínica (Manterola, 2001).

Respecto de la metodología utilizada vale la pena mencionar algunos hechos relevantes: En primer término, se definió como expertos a sujetos que podían realizar contribuciones válidas; es decir que poseen conocimientos basados en la práctica y experiencia actualizadas respecto de la materia (Kennedy; Price); optándose por un grupo heterogéneo ya que es fundamental la diversidad de puntos de vista, que otorga mayor interés y reflexión en los participantes (Powell). Esta estrategia, denominada técnica Delphi (Powell; Kennedy; Price), es un medio efectivo para generar consensos en un grupo, sin que los integrantes se reúnan físicamente. Se realiza a distancia contactando a los participantes a través de un cuestionario por correo tradicional o electrónico, a través del cual se realizan las diferentes ruedas de priorización de los ítems y dominios a valorar. Las ventajas de esta técnica son: que el anonimato de los aportes e ideas garantiza que cada una de ellas tenga el mismo valor e igual importancia en el análisis ulterior (Powell; Kennedy; Price); se elimina la influencia que podría ejercer sobre los demás el experto más persuasivo o de renombre, ya que todos los integrantes son de igual importancia; que la iteración entre los participantes es con una retroalimentación controlada y dirigida por un coordinador de modo que gradualmente la información irrelevante es eliminada (el intercambio de información entre los expertos no es libre); además, se permite una mayor franqueza en la expresión de las ideas de cada uno de los expertos, que en una reunión formal (Price).

A pesar de existir un instrumento que pudiera considerarse como similar a la propuesta actual, conocido como iniciativa STROBE, que ha sido ampliamente divulgada en diversas revistas, es importante hacer notar, que la Declaración STROBE está dirigida a artículos realizados con los tres diseños más importantes de la epidemiología analítica observacional: los estudios de cohortes, los estudios de casos y controles y los estudios transversales; sin embargo no considera los estudios más comunes, las series de casos y reportes de casos, como tampoco los estudios poblacionales y correlacionales; además de limitaciones que aparecen claramente mencionadas en el artículo (von Elm et al., 2007, 2008). 
A modo de conclusión se puede mencionar que se diseñó y construyó una pauta de chequeo que permite reportar resultados utilizando EOD; y que el instrumento generado pretende ser una ayuda no sólo a los investigadores que deciden reportar sus resultados a través de EOD; sino que también a los revisores y editores de revistas biomédicas que carecen de herramientas que de forma rápida y eficiente les permitan valorar este tipo de estudios.

MANTEROLA, C. \& ASTUDILLA, P. Checklist for reporting of descriptive observational studies. MINCIR Initiative.Int. J. Morphol., 31(1):115-120, 2013.

SUMMARY: Descriptive observational studies (DOE) constitute at least $70 \%$ of the published articles in various scientific journals in the field of surgery, and despite this, there are no instruments to guide writers and to assist reviewers in the result report with DOE. The aim of this study is to design and build a checklist for reporting results with EOD. Two-stage study with qualitative methodology. In a first stage, a proposal was designed, by collecting items and domains from an extensive review of the literature. In the second one, an instrument was developed by applying reduction items and domains through a panel of 45 experts in research methodology, clinical academics, reviewers and editors of biomedical journals, thereby applying facade and content validity. The items and domains incorporated into the final instrument were those which achieved over $80 \%$ agreement between the participants (36 of 45 ). In this way an instrument composed of 19 items and grouped into four domains was created. A checklist that will help to report the results using DOE was designed and built.

KEY WORDS: Observational studies; Epidemiologic Studies; Cross-Sectional Studies; Longitudinal Studies; Case series; Case Reports.

\section{REFERENCIAS BIBLIOGRÁFICAS}

Black, N. Why we need observational studies to evaluate the effectiveness of health care. BMJ, 312(7040):1215-8, 1996.

Bossuyt, P. M.; Reitsma, J. B.; Bruns, D. E.; Gatsonis, C. A.; Glasziou, P. P.; Irwig, L. M.; et al. The STARD statement for reporting studies of diagnostic accuracy: explanation and elaboration. Ann. Intern. Med., 138(1):W1-12, 2003.

Burgos, M. E. \& Manterola, C. Cómo interpretar un estudio sobre pruebas diagnósticas. Rev. Chil. Cir., 62(3):301-8, 2010.

Funai, E. F.; Rosenbush, E. J.; Lee, M. J. \& Del Priore, G. Distribution of study designs in four major US journals of obstetrics and gynecology. Gynecol. Obstet. Invest., 51(1):8-11, 2001.

Glasziou, P.; Vandenbroucke, J. P. \& Chalmers, I. Assessing the quality of research. BMJ, 328(7430):39-41, 2004.

Jones, J. \& Hunter, D. Consensus methods for medical and health services research. BMJ, 311(7001):376-80, 1995.

Kennedy, H. P. Enhancing Delphi research: methods and results. $J$. Adv. Nurs., 45(5):504-11, 2004.

Manterola, C. Estrategias de investigación. Diseños observacionales 1a parte. Estudios descriptivos. Rev. Chil. Cir., 53:229-33, 2001.

Manterola, C.; Busquets, J.; Pascual, M. \& Grande, L. What is the methodological quality of articles on therapeutic procedures published in Cirugía Española? Cir. Esp., 79(2):95-100, 2006a.
Manterola, C.; Pineda, V.; Vial, M.; Losada, H. \& MINCIR Group. Which is the methodological quality of human therapy studies in ISI surgical publications? Ann. Surg., 244(5):827-32, $2006 \mathrm{~b}$.

Manterola, C. \& Grande, L. Methodological quality of articles on therapeutic procedures published in cirugia española. Evaluation of the period 2005-2008. Cir. Esp., 87(4):244-50, 2010 .

Manterola, C. Introducción a los diseños de investigación. Los estudios observacionales. En: Introducción a la medicina basada en la evidencia. Burdiles, P. (edr.). Fundamentos del Cuidado Quirúrgico. Santiago de Chile, Mediterráneo, 2011. pp.462-73.

Moher, D.; Schulz, K.F. \& Altman, D.G. The CONSORT statement: revised recommendations for improving the quality of reports of parallel-group randomised trials. Lancet, 357(9263):1191-4, 2001.

Papanikolaou, P. N.; Christidi, G. D. \& Ioannidis, J. P. Comparison of evidence on harms of medical interventions in randomized and nonrandomized studies. CMAJ, 174(5):635-41, 2006.

Pineda, V.; Manterola, C.; Vial, M. \& Losada, H. ¿Cuál es la calidad metodológica de los artículos referentes a terapia publicados en la Revista Chilena de Cirugía? Rev. Chil Cir., 57(6):500-7, 2005.

Pope, C. \& Mays, N. Reaching the parts other methods cannot reach: an introduction to qualitative methods in health and health services research. BMJ, 311(6996):42-5, 1995. 
MANTEROLA, C. \& ASTUDILLO, P. Lista de verificación para el reporte de estudios observacionales descriptivos. Iniciativa MINCIR. Int. J. Morphol., 31(1):115-120, 2013.

Powell, C. The Delphi technique: myths and realities. J. Adv. Nurs., 41(4):376-82, 2003.

Price, B. Delphi survey research and older people. Nurs. Older People, 17(3):25-31, 2005.

Scales, C. D. Jr.; Norris, R. D.; Peterson, B. L.; Preminger, G. M. \& Dahm, P. Clinical research and statistical methods in the urology literature. J. Urol., 174(4 Pt. 1):1374-9, 2005.

Stroup, D. F.; Berlin, J. A.; Morton, S. C.; Olkin, I.; Williamson, G. D.; Rennie, D.; Moher, D.; et al., Meta-analysis of observational studies in epidemiology: a proposal for reporting. Meta-analysis of Observational Studies in Epidemiology (MOOSE) group. JAMA, 283(15):2008-12, 2000.

von Elm, E.; Altman, D. G.; Egger, M.; Pocock, S. J.; Gøtzsche, P. C. \& Vandenbroucke J. P. STROBE Initiative. The Strengthening the Reporting of Observational Studies in Epidemiology (STROBE) statement: guidelines for reporting observational studies. Ann. Intern. Med., 147(8):573-7, 2007.

von Elm, E.; Altman, D. G.; Egger, M.; Pocock, S. J.; Gøtzsche, P. C. \& Vandenbroucke J. P. Declaración de la Iniciativa STROBE (Strengthening the Reporting of Observational studies in Epidemiology): directrices para la comunicación de estudios observacionales. Gac. Sanit., 22(2):144-50, 2008.

\author{
Dirección para correspondencia: \\ Dr. Carlos Manterola \\ Departamento de Cirugía y Traumatología \\ Universidad de La Frontera \\ Casilla 54-D \\ Temuco \\ CHILE \\ Teléfono:56-45-325760 \\ Fax: $\quad 56-45-325761$ \\ E-mail: carlos.manterola@ufrontera.cl
}

Recibido : 04-11-2012

Aceptado: 04-12-2012 